\title{
Nucleic-acid analysis in new fields of metrology
}

\author{
Marjana Camloh $^{1, a}$, Tanja Dreo ${ }^{1}, K_{\text {Kristina Gruden }}{ }^{1}$, Nataša Mehle ${ }^{1}$, Mojca Milavec $^{1}$ and Jana Žel ${ }^{1}$ \\ ${ }^{1}$ Department of Biotechnology and Systems Biology, National Institute of Biology, Večna pot 111, SI-1000 Ljubljana, Slovenia
}

\begin{abstract}
Nucleic-acid analysis (NAA) represents a key bioanalysis to which metrological approaches are applied in various fields, such as biotechnology, food safety, public health, and environmental monitoring. NAA enables detection, identification and quantification of nucleic acids of different organisms and matrices. Approaches specific for the target are most common, and real-time polymerase chain reaction (qPCR) analysis is the most frequently used technique. Other methods are being developed that intensively address specific needs. For example, digital PCR enables sensitive detection and accurate absolute quantification without comparison to reference materials, and rapid isothermal approaches provide simple target detection on-site. At the National Institute of Biology, extensive research has been carried out over the last two decades for the development and assessment of NAA techniques and their applications to different fields. Here, we present some of our experiences with the application of metrological approaches to studies of genetically modified organisms and plant pathogenic microorganisms.
\end{abstract}

\section{Introduction}

Biological measurements (bioanalysis) have vital roles in different fields that affect quality of life. As in other fields, the aim is to ensure comparable measurements results with traceability to the International System of Units, when possible [1, 2]. The detection, identification and quantification of nucleic acids (nucleicacid analysis; NAA) represent one of the key bioanalysis. Accurate, reliable and traceable measurements of nucleic acids are crucial for meaningful application of life science research and development in the fields of biotechnology, food safety, health, pharmacy, medicine, environmental monitoring, and others.

The importance of bioanalysis is recognized internationally in metrology, as reflected in the establishment in 2002 of the Bioanalysis Working Group (BAWG) by the International Bureau of Weights and Measures (BIPM), in the framework of the Consultative Committee for Amount of Substance: Metrology in Chemistry and Biology (CCQM). The BAWG deals with bioanalysis of large macromolecules, and of biomolecular entities, where the target species (i.e., the analyte) is of biological origin in a biological measurement context [2]. Furthermore, the importance of bioanalysis was identified and supported also regionally during implementation of the European Metrology Research Programme (EMRP) by the European Association of National Metrology Institutes (EURAMET) through funding of metrological projects dealing with bioanalyses (e.g., the INFECTMET project "Metrology for monitoring infectious diseases, antimicrobial resistance, and harmful microoganisms", http://infectmet.lgcgroup.com/; the
BioSITrace project "Traceability for biologically relevant molecules and entities", http://biositrace.lgcgroup.com/). The same is expected for the European Metrology Programme for Innovation and Research, which followed on from the EMRP.

Increasing demands on the implementation of metrological concepts in bioanalysis are evident also in the latest edition of the International Vocabulary of Metrology [3], where an attempt has been made to meet conceptual needs of measurement in fields such as biochemistry, food science, forensic science, and molecular biology.

In the field of NAA, rapid technological advances have occurred over the last few decades, and efforts have been made to define key measurement parameters in NAA. However, there remain challenges that are as yet not resolved. The major challenges include the absence of certified reference materials (CRMs), and sometimes undefined validation parameters and unclear use of units in reporting results in certain fields. Moreover, reference / standard methods, key comparison studies, and proficiency testing schemes are not always available.

\section{Bioanalysis expertise of the National Institute of Biology}

The National Institute of Biology (NIB) has been a holder of a national standard (often referred to as a 'Designated Institute' by the EURAMET) in the field of metrology, in chemistry/ amounts of substance, since 2009. Metrological approaches are deeply integrated into the research and development work at the NIB. To ensure

marjana.camloh@nib.si 
accurate and reliable measurements, this often includes activities towards the initial application of metrological approaches for a particular method; e.g., real-time PCR (qPCR) for qualitative and quantitative detection, digital (d)PCR, LAMP, and others. In the past, methods arising from technological innovations were often used for years before the development and wider application of standardised approaches. This situation is, however, now improving because of increased awareness of researchers for the need for standardised critical measurement parameters and their influence on the interpretation of results. At the moment, various networks and associations are working towards a common goal of rapid standardisation and control of critical measurement parameters. While such critical parameters are addressed both during the validation and implementation of the methods, the approaches are often limited to a certain field and are not always well integrated into existing metrological approaches and synchronised across fields of application. This inconsistent approach can lead to substantial discrepancies in the analysis and interpretation of results obtained between fields.

At the NIB, intensive research has been carried out over the last two decades on the development and assessment of bioanalysis based on amplification of nucleic acids and their application in different fields, and in particular for genetically modified organisms (GMOs) and plant pathogens. We have observed that, depending on the aim within a particular field, or even of a particular analysis, the requirements of the method performance can vary considerably. This in turn leads to different, specialised, approaches to assess the performances of the methods in different fields.

Here, we describe our experience with bioanalysis in the fields of GMOs and plant pathogens. We address both the current status and the critical shortfalls that will require our coordinated efforts over the next few years.

\subsection{Genetically modified organisms}

GMOs are organisms in which the genetic material has been altered in a way that does not occur naturally, through the application of gene technology. A GMO is formed by the insertion of one or more functional genes (e.g., the association of two or more DNA sequences arising from different species) into the genome of an organism, to produce new genetic combinations (events) that are of value to science, medicine, agriculture and industry (reviewed in [4]).

The labelling of GMOs is mandatory in the European Union (EU). However, GMO labelling requirements do not apply to food and feed that contain approved GM events below a threshold of $0.9 \%$ of the food or feed ingredients when considered individually, if this presence is adventitious or technically not avoidable [5].

Accurate, reliable and traceable quantification of GMOs are a prerequisite for the traceability of GMOs in the food chain, from the field to the final consumer, as are appropriate labelling of food and feed products. Therefore, for GMOs, the NIB focus has been on accurate and reliable quantification of DNA targets using qPCR, which has been recognized as the golden standard for GMO detection and quantification for many years (summarised in [6]).

As laboratories have been facing many challenges during the adoption of GMO testing, the European Network of GMO Laboratories (ENGL) was established in 2002. The NIB actively participates in several working groups, which produced key guidance documents to instruct GMO laboratories on different aspects of GMO detection; e.g., minimum performance requirements for methods development $[7,8]$, implementation of validated methods in the laboratory [9], and calculation of measurement uncertainty [10]. The NIB has also participated in the development of international standards on GMO detection at the level of CEN and ISO.

Nowadays, there are novel GMOs being brought to the market, that are both approved or are in the application process in the EU after being approved elsewhere [11]. The increased number of GMOs and the lower non-compliance limit of $0.1 \%$ require more efficient, high throughput, affordable approaches for GMO detection. A multiplex qPCR screening method that was developed and validated by the NIB in collaboration with the Federal Office of Consumer Protection and Food Safety, Germany, has addressed some of these issues and is now used in many laboratories [12]. Additionally, new techniques have been examined for their potential in GMO detection; e.g., NAIMA and LAMP [13, 14].

In the past few years, dPCR has opened new perspectives in GMO detection. At the NIB, we have focused on droplet dPCR, which enables absolute quantification without standard curves, avoids amplification efficiency bias associated with qPCR, allows more accurate estimations of targets at low copy numbers, and in combination with multiplexing, significantly improves cost efficiency. We have developed a method for detection and quantification of MON810 maize [15]. Recently, high-multiplex (10-plex and 5-plex) dPCR methods were also developed that cover 12 EU approved GM maize [16].

There is still ongoing debate on the measurement unit for GMOs, because different GMO regulations have set different measurement units, or the measurement unit is not explicitly specified. So there is the possibility to express the GMO content as: (i) percentage of eventspecific DNA copy numbers (e.g., MON810) in relation to the target taxon-specific DNA copy numbers (e.g., hmg-genetic element specific for maize), calculated in terms of haploid genomes, as has been set by [17]; or (ii) mass fractions, as the majority of CRMs are certified for the mass fraction. dPCR has also enabled evaluation of the ratio between the copy numbers of DNA and the mass fractions. At the NIB, different reference materials have been characterised in the framework of the GMOval project (http://www.gmoval.com/).

To date, only one key comparison study for GMOs has been completed, which was organised in the framework of the CCQM (piloted by the Institute for Reference Materials and Methods, in Geel, Belgium). This study focussed on the measurement of MON810 (CCQM-K86). An additional key comparison on BT63 rice (CCQM-K86.b) was recently initiated. The accurate 
results obtained by the NIB on CCQM-K86 enabled the first NIB Calibration and Measurement Capability (CMC) entry for MON810/hmg, in the BIPM key comparison database.

There is still open discussion in terms of whether CMC entries for GMOs should be for individual GMOs or whether one should cover all GMOs of the same species (Table 1).

\subsection{Microorganisms - plant pathogens}

The term plant pathogen includes bacteria, fungi, viruses, viroids and other organisms that can cause diseases in plants. Many of these cannot be efficiently managed with pesticides, and their control relies on the exclusion of infected plant material, as identified through laboratory testing. Some plant pathogens present high risk. The quarantine bacteria Ralstonia solanacearum and Clavibacter michiganensis subsp. sepedonicus are the causative agents of potato brown rot and ring rot, respectively, and they represent direct risk to the production of potatoes, which are an important staple food where the global yearly production exceeds 370 million metric tons [18]. To prevent the spread of these pathogens in Europe, a monitoring system is in place that includes visual inspections of potato fields, random sampling of potato tubers and other host plants, and laboratory testing of tubers for latent infection, for both of these pathogens, and using methods prescribed at the level of the European Commission [19, 20]. In the case of a positive result, phytosanitary measures are applied. This mainly involves the destruction of all infected plant material, which can easily reach hundreds of tons, with disinfection of the agricultural machinery and storage facilities, and also restrictions on planting in the contaminated fields in subsequent years. To minimise false-positive results that might lead to considerable and unjustified costs, methods are usually used in combination, and thus NAA is one of the methods used. For some pathogens, however, NAA is the only option available, and this needs to be especially carefully controlled to provide accurate results.

In Europe, the European and Mediterranean Plant Protection Organisation (EPPO) was founded as early as 1955, and it covers different areas of plant protection. The EPPO is a key player in the harmonisation of approaches taken by laboratories to evaluate and validate analyses, through the development of widely accepted guidelines. A set of general EPPO guidelines [21, 22] defines the minimum parameters that need to be determined, along with an agreed experimental approach for their determination. The EPPO guidelines [23] further serve to aid in the interpretation of the ISO/IEC 17025 standard in the plant health field, where CRMs with defined target levels/concentrations are not available, and the availability of interlaboratory comparison studies is limited. In addition, recently EPPO guidelines on organization of interlaboratory comparisons were also published [24]. The NIB knowledge and experience in validation, identification and detection of several plant pathogens has been actively transferred to the development of standards through cooperation with different EPPO expert panels.

At the NIB, qPCR-based amplification methods for detection and identification of plant pathogens have been developed, validated and published. Also, in parallel, we have developed approaches for evaluation, interpretation and reporting on qPCR. qPCR for detection of a quarantine grapevine pathogen, Xylophilus ampelinus [25], was the first qPCR-based technique included in the EPPO diagnostic protocols, and it is still in use today [26]. Other qPCR methods that have been developed at the NIB can detect plant pathogenic viruses (e.g., potato virus $\mathrm{Y}$ isolates, Pepino mosaic virus [27]) and bacteria (e.g., Erwinia amylovora), and Flavescence dorée and Bois noir phytoplasma in grapevine [28, 29]. Recently, methods using dPCR for detection and quantification of microorganisms have been published [30, 31]. Additionally, LAMP isothermal method was developed for rapid and cost-effective detection of microorganisms $[32,33]$.

For quarantine plant pathogens, a zero-tolerance concept is in place; i.e., there is no acceptable threshold defined, but we aim to detect as low a target concentration as possible, and the target concentration rarely has consequences for the measures taken. Consequently, although most tests that are used are qualitative, and thus provide plus/ minus results, they need to be developed and evaluated as quantitative tests that can focus on the lower range of target concentrations [34]. This stresses the importance of differentiating background signals from true positives that has led to several approaches proposed for $\mathrm{qPCR}$ and $\mathrm{APCR}$ that are based on equal distributions of risk to producer and user [35], and on the assessment of the probability of false-positive results from the validation data [36].

Contrary to the GMO field, where the validation approach is modular, the validation in plant pathology is carried out for an application of a certain test (e.g., qPCR) for detection of a certain organism/target (e.g., $R$. solanacearum) in a certain matrix (e.g., potato tubers). To use the same test for the detection of $R$. solanacearum in water, the method needs to be carefully re-examined, especially taking into account measurement uncertainties at different levels [23], and if additional experiments need to be performed to confirm its suitability for the new matrix. Considering the large number of different organisms (European legislation lists more than 200 different pathogens) and the even greater number of matrices, the non-modular approach to validation necessitates an enormous effort to be dedicated to validation of methods in plant health. While EPPO supports open sharing of the validation data through scientific publications or validation reports deposited with an on-line database (http://dc.eppo.int/validationlist.php), and Euphresco ERA-NET project does cover validation and interlaboratory comparisons, funding limitations still present a real obstacle to validation of larger numbers of methods.

The absence of certified (or other) reference materials with defined levels of targets requires that laboratories themselves prepare in-house controls for both validation and routine testing. A significant advance in recent years 
has been the use of dPCR, which allows absolute target quantification and can be used for characterisation of these in-house controls (Table 1).

Table1. Some of the measurement parameters and documents considered at the NIB during NAA validation/verification and analysis of GMOs and microorganisms - plant pathogens using qPCR methodologies.

\begin{tabular}{lll}
\hline $\begin{array}{l}\text { Measurement } \\
\text { parameters, documents }\end{array}$ & Genetically modified organisms & Microorganisms - plant pathogens \\
\hline $\begin{array}{l}\text { Type of samples } \\
\text { (organisms, matrices) }\end{array}$ & $\begin{array}{l}\text { Foodstuffs, feed, seed, plants and agricultural } \\
\text { products. }\end{array}$ & $\begin{array}{l}\text { Plants, insects, environmental samples (water, } \\
\text { soil). }\end{array}$ \\
\hline $\begin{array}{l}\text { Measu } \\
\text { rand } \quad \text { Qualyte }\end{array}$ & $\begin{array}{l}\text { Target DNA sequences. } \\
\text { Mass fractions or DNA copy number ratio. }\end{array}$ & $\begin{array}{l}\text { Target DNA or RNA sequences. } \\
\text { Qualitative result reporting the presence or } \\
\text { absence of the target DNA/RNA in an } \\
\text { analysed sample. }\end{array}$ \\
\hline
\end{tabular}

\begin{tabular}{ll}
\hline Measurement unit & Defined and used mostly in two ways: \\
& Mass fraction of a GMO expressed in \\
& percentage [11], or the percentage of event- \\
& specific DNA copy numbers in relation to the \\
& target taxon-specific DNA copy numbers, \\
& calculated in terms of haploid genomes [17]. \\
\hline Standard measurement & ISO standards on GMOs, internal procedures \\
procedure & mostly based on validated methods of the \\
& European Union Reference Laboratory for \\
& Genetically Modified Food and Feed.
\end{tabular}

Target DNA/RNA.

\begin{tabular}{ll}
\hline Measurement result & $\begin{array}{l}\text { Measured value of nucleic acids originating } \\
\text { from GMOs and a measurement uncertainty. }\end{array}$ \\
\hline
\end{tabular}

Measurement uncertainty Top down [10].

approach

\begin{tabular}{ll}
\hline Certified reference & Mostly available from the Institute for \\
materials & Reference Materials and Methods, and the \\
& American Oil Chemists' Society.
\end{tabular}

Method validation $\quad$ Modular [7,8].
approach

\begin{tabular}{|c|c|c|}
\hline $\begin{array}{l}\text { Validation/verification } \\
\text { guidelines }\end{array}$ & ENGL document [9]. & EPPO documents $[21,22]$. \\
\hline Validated methods & $\begin{array}{l}\text { Mostly available on: } \underline{\text { http://gmo- }} \\
\text { crl.jrc.ec.europa.eu/StatusOfDossiers.aspx. }\end{array}$ & $\begin{array}{l}\text { Validation data available for some methods } \\
\text { (http://dc.eppo.int/validationlist.ph; scientific } \\
\text { publications). }\end{array}$ \\
\hline Key comparisons & Rarely available at CCQM (from 2009 on). & Not available. \\
\hline Inter-laboratory studies & $\begin{array}{l}\text { Organised by the European Union Reference } \\
\text { Laboratory for Genetically Modified Food and } \\
\text { Feed; the International Seed Testing } \\
\text { Association; the United States Department of } \\
\text { Agriculture, Grain Inspection, Packers and } \\
\text { Stockyards Administration. }\end{array}$ & $\begin{array}{l}\text { Rarely organised in the frame of different } \\
\text { projects (e.g., Euphresco), not available for } \\
\text { many tests and matrices. }\end{array}$ \\
\hline $\mathrm{CMC}$ & MON810/hmg. & / \\
\hline
\end{tabular}




\subsection{Conclusions}

For NAA, many challenges still need to be addressed, and the metrological aspects need to be investigated further. These are often dependant on the field of application, as we have presented given only two examples of GMOs and plant pathogens.

We would like to emphasise that it is very important to share knowledge and experience from one field to another. An effective example of this is the participation of the NIB in EMRP projects such as "Metrology for monitoring infectious diseases, antimicrobial resistance, and harmful micro-oganisms" (INFECT-MET) and "Traceability for biologically relevant molecules and entities" (BioSITrace). Within these projects, the experience and approaches from different fields are combined for the benefit of all partners, and these can lead to optimal, harmonised applications of metrological approaches. Thus, a relatively small Designated Institute such as the NIB can contribute significantly to the specialised fields of metrology, and can share the knowledge on NAA across wider metrological aspects.

\section{References}

1. http://www.bipm.org/metrology/chemistry-biology/

2. http://www.bipm.org/en/committees/cc/wg/bawg.ht $\underline{\mathrm{ml}}$

3. JCGM 200:2012(E/F) (2012)

4. M. Milavec, D. Dobnik, L. Yang, D. Zhang, K. Gruden, J. Žel, Anal. Bioanal. Chem. 406, 26 (2014)

5. Directive 1830/2003/EC. Off. J. Eur. Union 268 (2003)

6. J. Žel, M. Milavec, D. Morisset, D. Plan, G. Van den Eede, K. Gruden, How to Reliably Test for GMOs, Springer, New York (2012)

7. ENGL, Definition of minimum performance requirements for analytical methods of GMO testing (2008).

8. ENGL, JRC95544 (2015)

9. ENGL, EUR 24790 EN (2011)

10. S. Trapmann, M. Burns, H. Broll, R. Macarthur, R. Wood, J. Žel, JRC50052 (2009)

11. European Union Regulation $N^{\circ} 619 / 2011$ (2011).

12. I. Huber, A, Block, D. Sebah, F. Debode, D. Morisset, et al. J. Agric. Food Chem. 61, 43 (2013).
13. D. Morisset, D. Dobnik, S. Hamels, J. Žel, K. Gruden, Nucl. Acids Res. 36, 18:e118. (2008)

14. G.J. Randhawa, M. Singh, D. Morisset, P. Sood, J. Žel, J. Agric. Food Chem. 61, 47 (2013).

15. D. Morisset, D. Štebih, M. Milavec, K. Gruden, J. Žel, PLoS ONE 8, 5:e62583 (2013)

16. D. Dobnik, B. Spilsberg, A. Bogožalec, A. HolstJensen, J. Žel, Anal. Chem. (in press)

17. European Union Recommendation 2004/787/EC

18. http://faostat3.fao.org/browse/ranking

19. Council directive $98 / 57 /$ EC OJ L 235 (1998)

20. Council directive 93/85/EEC OJ L 259 (1933)

21. EPPO PM 7/76 EPPO Bulletin 44, 3 (2014)

22. EPPO PM 7/84, EPPO Bulletin 37 (2007)

23. EPPO PM 7/98, EPPO Bulletin 44, 2 (2014)

24. EPPO PM 7/122, EPPO Bulletin 44, 3 (2014)

25. T. Dreo, K. Gruden, C. Manceau, J.D. Janse, M. Ravnikar, Plant Pathol. 56 (2007)

26. EPPO PM 7/96, EPPO Bulletin, 39 (2009)

27. I.Gutierrez-Aguirre, N. Mehle, D. Delić, K. Gruden, R. Mumford, M. Ravnikar, J. Virol. Methods, 162, 12 (2009)

28. M. Pirc, M. Ravnikar, J. Tomlinson, T. Dreo, Plant Pathol. 58 (2009)

29. M. Hren, J. Boben, A. Rotter, P. Kralj, K. Gruden, M. Ravnikar, Plant Pathol. 56 (2007)

30. T. Dreo, M. Pirc, Ž. Ramšak, J. Pavšič, M. Milavec, J. Žel, K. Gruden, Anal. Bioanal. Chem. 406 (2014)

31. N. Mehle, T. Dreo, M. Ravnikar, Phytopathogenic mollicutes, 4, 1 (2014)

32. R. Lenarčič, D. Morisset, N. Mehle, M. Ravnikar, Plant Pathol. 62, 5 (2013)

33. P. Kogovšek, J. Hodgetts, J. Hall, N. Prezelj, P. Nikolić, N. Mehle, et al. Plant Pathol. 64, 2 (2015)

34. S.A. Bustin, V. Benes, J.A. Garson, J. Hellemans, J. Huggett, M. Kubista, et al. Clin. Chem. 55, 4 (2009)

35. A.Chandelier, K. Ivors, M. Garbelotto, J. Zini, F. Laurent, M. Cavelier, EPPO Bulletin, 36 (2006)

36. C.H. Roberts, A. Last, S. Molina-Gonzalez, E. Cassama, R. Butcher, M. Nabicassa, et al. J. Clin. Microbiol. 51 (2013)

\section{Acknowledgements}

This study was co-financed by the Metrology Institute of the Republic of Slovenia (Contract $\mathrm{N}^{\circ}$ 640118/2008/67). INFECT MET and BioSITrace are EMRP projects that are jointly funded by the EMRP participating countries within the EURAMET and the EU. 